\title{
Reducing population stratification bias: stratum matching is better than exposure
}

\author{
Wen-Chung Lee ${ }^{\mathrm{a}, \mathrm{b}, *}$, Liang-Yi Wang, \\ ${ }^{a}$ Research Center for Genes, Environment and Human Health, College of Public Health, National Taiwan University, Taiwan \\ ${ }^{\mathrm{b}}$ Graduate Institute of Epidemiology, College of Public Health, National Taiwan University, Taiwan \\ Received 1 October 2007; received in revised form 4 February 2008; accepted 29 February 2008
}

\begin{abstract}
Objective: Genetic studies of complex human diseases rely heavily on the epidemiologic association paradigm, particularly the population-based case-control designs. This study aims to compare the matching effectiveness in terms of bias reduction between exposure matching and stratum matching.

Study Design and Setting: Formulas for population stratification bias were derived. An index of matching effectiveness was constructed to compare the two types of matching.

Results: It was found that exposure matching can paradoxically increase the magnitude of population stratification bias sometimes, whereas stratum matching can guarantee to reduce it.

Conclusion: The authors propose two simple rules for genetic association studies: (a) to match on anything that helps to delineate population strata such as race, ethnicity, nationality, ancestry, and birthplace and (b) to match on an exposure only when it is a strong predictor of the disease and is expected to have great variation in prevalence across population strata. (c) 2008 Elsevier Inc. All rights reserved.
\end{abstract}

Keywords: Case-control study; Epidemiologic methods; Genetic association study; Matching; Bias; Genetic epidemiology

\section{Introduction}

Genetic studies of complex human diseases rely heavily on the epidemiologic association paradigm, particularly the population-based case-control designs [1,2]. However, a case-control genetic association study may suffer from the "population-stratification bias" if it is conducted in a "stratified population" [3,4]. (The study population is "stratified," if it is composed of two or more strata in which gene frequencies and background disease rates differ across the strata.) Wacholder et al. [5] had done a theoretical and empirical study on the degree of population-stratification bias. Although they found the bias to be small in U.S. studies of cancer among nonHispanic Caucasians of European origin, they did emphasize the importance of appropriate design for genetic association studies.

"Matching" is a design method frequently used in epidemiology [6]. For the present context of a genetic association study, it is important to distinguish two types of

* Corresponding author. Graduate Institute of Epidemiology, College of Public Health, National Taiwan University, Room 536, Number 17, Xuzhou Road, Taipei 100, Taiwan. Tel.: +866-2-33228036; Fax: +8862-23511955.

E-mail address: wenchung@ntu.edu.tw (W.-C. Lee). matching, that is, the "exposure matching" and the "stratum matching." The exposure matching aims to match the cases and the controls on the risk factor profile (such as on sex, age, and/or on the exposure status of smoking, drinking, betel nut chewing, hepatitis B surface antigen). Whereas the purpose of a stratum matching is to ensure that the cases and their matched controls are coming from the same population stratum (by matching on stratumdelineating variables such as race, ethnicity, nationality, ancestry, and birthplace)[7].

However, the effects of these two types of matching in terms of bias reduction had not been studied. The environmental exposures, though they may be risk factors for the disease, are not associated with the gene under study in any given population stratum. Thus, one may argue that, if the stratum can be perfectly matched, the environmental exposures themselves cannot confound the study results and thus there is no point matching on them anymore [7]. But on the other hand, stratum matching is seldom perfect. The stratum-delineating variables used in a study are proxy at best for the population strata [7]. One cannot expect them to resolve the true stratum-membership for each and every subject in a study. To match on exposure or to match on stratum is thus a dilemma. 
In this study, we will first show using a hypothetical example that, contrary to popular belief, an exposure matching in a genetic association study can increase the magnitude of bias instead of decreasing it. We will then derive the mathematical formula for population-stratification bias and make a formal comparison about the matching effectiveness (ME) of exposure matching and stratum matching under various conditions. Note that in this study we focus on bias reduction. We will not address the issue of using matching to achieve greater precision.

\section{Bias increased by exposure matching: an observation}

Table 1 presents a hypothetical population, which is composed of two strata. Within either stratum, the gene (two types: high risk and low risk) and the exposure (two status: exposed and unexposed) are independent. The table also presents the number of cases for a particular disease. In any given stratum, the disease risk for those with the highrisk gene is three times that for those with the low-risk gene; and the disease risk for the exposed subjects is two times that for the unexposed. We assume that the presence of stratification in this hypothetical population is unknown to researchers. (This is often true in real practice, because race and ethnicity of a person are difficult to characterize using questionnaire. And there may be subethnicities within an ethnicity that make a correct identification even more difficult. And worse, population stratification is not limited

Table 1

A hypothetical population composed of two strata

\begin{tabular}{lcc}
\hline & Population number & Case number \\
\hline Stratum 1 & & \\
Exposed & & 96 \\
$\quad$ High-risk gene & 160,000 & 8 \\
Low-risk gene & 40,000 & \\
Unexposed & & 192 \\
High-risk gene & 640,000 & 16 \\
Low-risk gene & 160,000 & \\
& & \\
Stratum 2 & & 24 \\
Exposed & 160,000 & 32 \\
High-risk gene & 640,000 & \\
Low-risk gene & & 3 \\
Unexposed & 40,000 & 4 \\
High-risk gene & 160,000 & \\
Low-risk gene & & 120 \\
The entire population & & 40 \\
Exposed & & \\
High-risk gene & 320,000 & 20 \\
Low-risk gene & 680,000 & \\
Unexposed & & \\
High-risk gene & 680,000 & \\
Low-risk gene & 320,000 & \\
\hline
\end{tabular}

to known racial/ethnic groups. It may just be the results of incomplete admixing of populations because of subtle geographical, social, or political barriers.)

Table 2 presents the expected cell counts of the $2 \times 2$ table for an unmatched case-control study conducted in this population (with equal number of cases and controls). The odds ratio (relating the gene to the disease) is estimated as $\mathrm{OR}=(315 \times 187.5) /(187.5 \times 60)=5.25$, which is $(5.25-3) / 3=75 \%$ higher than its true value of 3 . (Note that the disease risk is so low that the "odds ratio" and the "relative risk" are essentially the same in this population.) Whereas, Table 3 presents the expected cell counts of the pair-matched table for a case-control study with exposure matching conducted in this population (1:1 matching). The odds ratio is now estimated as $\mathrm{OR}=144 / 26.4=5.45$, which is $(5.45-3) / 3=81.8 \%$ higher than the true value.

We see quite unexpectedly from this example that the exposure matching, instead of decreasing the magnitude of bias, can actually increase it! Note that here we perform a matched analysis for the matched design and yet still observe the bias to increase thereafter. This is in sharp contrast to the classical textbook examples where the bias incurred by matching in the design stage can be completely resolved by performing a matched data analysis or a conditional logistic regression [6].

\section{Formula for population-stratification bias}

It is assumed that the study population is a stratified population with a total of $J$ strata $(j=1,2, \ldots, J)$. Let $q_{j}$ denote the gene frequency $g_{j}=q_{j} /\left(1-q_{j}\right)$, the gene frequency odds), $b_{j}$, the background disease rate, and $m_{j}$, the total number of subjects (or more precisely, the total person-

Table 2

An unmatched case-control study (375 cases and 375 controls) in the hypothetical population of Table 1

\begin{tabular}{lcc}
\hline & Cases & Controls \\
\hline High-risk gene & $315^{\mathrm{a}}$ & $187.5^{\mathrm{c}}$ \\
Low-risk gene & $60^{\mathrm{b}}$ & $187.5^{\mathrm{d}}$ \\
\hline
\end{tabular}

Shown in entries are the expected numbers of subjects.

a Total number of cases in Table 1 with high-risk gene: $120+195=315$.

b Total number of cases in Table 1 with low-risk gene: $40+20=60$.

${ }^{c}$ Expected number of controls with high-risk gene:

$$
\begin{aligned}
& 375 \times \frac{\text { Population number in Table } 1 \text { with high-risk gene }}{\text { Total population number in Table } 1} \\
= & 375 \times \frac{320,000+680,000}{320,000+680,000+680,000+320,000}=187.5 .
\end{aligned}
$$

${ }^{\mathrm{d}}$ Expected number of controls with low-risk gene:

$$
\begin{aligned}
& 375 \times \frac{\text { Population number in Table } 1 \text { with low-risk gene }}{\text { Total population number in Table } 1} \\
= & 375 \times \frac{680,000+320,000}{320,000+680,000+680,000+320,000}=187.5 .
\end{aligned}
$$


Table 3

An exposure-matched case-control study (1:1 matching, a total of 375 matched pairs) in the hypothetical population of Table 1

\begin{tabular}{lll}
\hline & \multicolumn{2}{c}{ Controls } \\
\cline { 2 - 3 } Cases & High-risk gene & Low-risk gene \\
\hline High-risk gene & $171^{\mathrm{a}}$ & $144^{\mathrm{c}}$ \\
Low-risk gene & $26.4^{\mathrm{b}}$ & $33.6^{\mathrm{d}}$ \\
\hline
\end{tabular}

Shown in entries are the expected numbers of matched pairs.

${ }^{a}$ Number of exposed pairs with the case and the control both having the high-risk gene + number of unexposed pairs with the case and the control both having the high-risk gene $=120 \times 320,000 /(320,000+$ $680,000)+195 \times 680,000 /(680,000+320,000)=171$.

b Number of exposed pairs with the case having the low-risk gene while the control, high-risk gene + number of unexposed pairs with the case having the low-risk gene while the control, high-risk gene $=40 \times$ $320,000 /(320,000+680,000)+20 \times 680,000 /(680,000+320,000)=$ 26.4 .

c Number of exposed pairs with the case having the high-risk gene while the control, low-risk gene + number of unexposed pairs with the case having the high-risk gene while the control, low-risk gene $=120 \times 680,000 /$ $(320,000+680,000)+195 \times 320,000 /(680,000+320,000)=144$.

d Number of exposed pairs with the case and the control both having the low-risk gene + number of unexposed pairs with the case and the control both having the low-risk gene $=40 \times 680,000 /(320,000+$ $680,000)+20 \times 320,000 /(680,000+320,000)=33.6$.

time observations), in the $j$ th stratum. Let $\mathrm{RR}_{\mathrm{G}}$ denote the relative rate of disease for those with the gene as compared to those without. In this population as a whole, the disease rate for those with the gene is $R_{1}=\sum_{j} m_{j} q_{j} b_{j} \mathrm{RR}_{\mathrm{G}} / \sum_{j} m_{j} q_{j}$, and the disease rate for those without is $R_{0}=\sum_{j} m_{j}\left(1-q_{j}\right) b_{j} / \sum_{j} m_{j}\left(1-q_{j}\right)$.Thus the "confounding rate ratio" (CRR) (defined as the ratio of the biased relative rate and the true relative rate) [8] is $\mathrm{CRR}^{\mathrm{B}}=$ $\left(R_{1} / R_{0}\right) / \mathrm{RR}_{\mathrm{G}}=\sum_{j} w_{j} g_{j} b_{j} /\left(\sum_{j} w_{j} g_{j}\right) \times\left(\sum_{j} w_{j} b_{j}\right)$, where $w_{j}=m_{j}\left(1-q_{j}\right) / \sum_{k} m_{k}\left(1-q_{k}\right)$ is the "weight" used in the above summation. Note that we use the superscript " $\mathrm{B}$ " in CRR to specify that the confounding is due to the stratum background rates.

Further, we let $\bar{\varphi}_{\mathrm{G}}=\sum_{j} w_{j} g_{j}$ and $\bar{\varphi}_{\mathrm{B}}=\sum_{j} w_{j} b_{j}$ denote the means, and $\mathrm{SD}\left(\varphi_{\mathrm{G}}\right)=\sqrt{\sum_{j} w_{j}\left(g_{j}-\bar{\varphi}_{\mathrm{G}}\right)^{2}} \quad$ and $\mathrm{SD}\left(\varphi_{\mathrm{B}}\right)=\sqrt{\sum_{j} w_{j}\left(b_{j}-\bar{\varphi}_{\mathrm{B}}\right)^{2}}$, the standard deviations, of the gene frequency odds and the background rates, respectively. It is of interest to note that the relative bias (RB) (defined as the ratio of the bias and the true relative rate) can be expressed as

$$
\mathrm{RB}^{\mathrm{B}}=\mathrm{CRR}^{\mathrm{B}}-1=r_{\mathrm{GB}} \times \mathrm{CV}_{\mathrm{G}} \times \mathrm{CV}_{\mathrm{B}},
$$

where $r_{\mathrm{GB}}=\left[\sum_{j} w_{j}\left(g_{j}-\bar{\varphi}_{\mathrm{G}}\right)\left(b_{j}-\bar{\varphi}_{\mathrm{B}}\right)\right] /\left[\mathrm{SD}\left(\varphi_{\mathrm{G}}\right) \times \mathrm{SD}\left(\varphi_{\mathrm{B}}\right)\right]$ is the correlation coefficient between gene frequency odds and background rates, $\mathrm{CV}_{\mathrm{G}}=\mathrm{SD}\left(\varphi_{\mathrm{G}}\right) / \bar{\varphi}_{\mathrm{G}}$, the coefficient of variation in gene frequency odds, and $\mathrm{CV}_{\mathrm{B}}=$ $\operatorname{SD}\left(\varphi_{\mathrm{B}}\right) / \bar{\varphi}_{\mathrm{B}}$, the coefficient of variation in background rates, among the total of $J$ strata. It is clear from the above formula for relative bias that there would be no population stratification bias, if (a) the gene frequency odds and the background rates are uncorrelated, (b) there is no variation in the gene frequency odds, or (c) there is no variation in the background rates. Also, one notices that overestimation would occur when the gene frequency odds and the background rates are positively correlated, and underestimation would occur when negatively correlated.

Further assume that beyond the stratum background rates, there is a certain "exposure" that may also confound the results. The exposure has a relative rate of $\mathrm{RR}_{\mathrm{E}}$ (we assumed $R_{E} \geqslant 1$ ). Within any given stratum, the gene and the exposure are independent (note that in the population as a whole, they may be not) [9]. The exposure has prevalence of $p_{j}$, and exposure prevalence odds of $e_{j}=p_{j} /\left(1-p_{j}\right)$, in the $j$ th stratum. The exposure prevalence odds has a mean of $\bar{\varphi}_{\mathrm{E}}=\sum_{j} w_{j} e_{j}$ and a standard deviation of $\operatorname{SD}\left(\varphi_{\mathrm{E}}\right)=$ $\sqrt{\sum_{j} w_{j}\left(e_{j}-\bar{\varphi}_{\mathrm{E}}\right)^{2}}$. Under this setting, the disease rate for those without the gene in the $j$ th stratum is $b_{j}^{*}=p_{j} \mathrm{RR}_{\mathrm{E}} b_{j}+\left(1-p_{j}\right) b_{j}$, and the confounding rate ratio is $\mathrm{CRR}^{\mathrm{B}, \mathrm{E}}=\sum_{j} w_{j} g_{j} b_{j}^{*} /\left(\sum_{j} w_{j} g_{j}\right) \times\left(\sum_{j} w_{j} b_{j}^{*}\right)$ (the superscript "B,E" denotes that the confounding may be due to the stratum background rates and the exposure prevalence odds).

Using the delta method (assuming $\mathrm{RR}_{\mathrm{E}} \rightarrow 1$ ) [10], it can be shown that the relative bias is now composed of two terms:

$$
\begin{aligned}
& \mathrm{RB}^{\mathrm{B}, \mathrm{E}} \approx \mathrm{RB}^{\mathrm{B}}+\mathrm{RB}^{\mathrm{E}} \\
& =r_{\mathrm{GB}} \times \mathrm{CV}_{\mathrm{G}} \times \mathrm{CV}_{\mathrm{B}}+f \times r_{\mathrm{GE}} \times \mathrm{CV}_{\mathrm{G}} \times \mathrm{CV}_{\mathrm{E}},
\end{aligned}
$$

where $f=\left[\left(\mathrm{RR}_{\mathrm{E}}-1\right) \bar{\varphi}_{\mathrm{E}}\right] /\left[\left(\mathrm{RR}_{\mathrm{E}} \bar{\varphi}_{\mathrm{E}}+1\right)\left(\bar{\varphi}_{\mathrm{E}}+1\right)\right]$ is a factor depending on $\mathrm{RR}_{\mathrm{E}}$ and $\bar{\varphi}_{\mathrm{E}}, \quad r_{\mathrm{GE}}=\left[\sum_{j} w_{j}\left(g_{j}-\bar{\varphi}_{\mathrm{G}}\right)\right.$ $\left.\left(e_{j}-\bar{\varphi}_{\mathrm{E}}\right)\right] /\left[\mathrm{SD}\left(\varphi_{\mathrm{G}}\right) \times \mathrm{SD}\left(\varphi_{\mathrm{E}}\right)\right]$, the correlation coefficient between gene frequency odds and exposure prevalence odds, and $\mathrm{CV}_{\mathrm{E}}=\mathrm{SD}\left(\varphi_{\mathrm{E}}\right) / \bar{\varphi}_{\mathrm{E}}$, the coefficient of variation in exposure prevalence odds, among the total of $J$ strata.

\section{Effects of matching}

If a study matches on exposure (assuming that exposure status for every study subjects can be measured without error), the $\mathrm{RB}^{\mathrm{E}}$ term will vanish and the bias will be solely from the $R^{B}{ }^{B}$ term. That is,

$$
\mathrm{RB} \text { (for exposure matching }) \approx \mathrm{RB}^{\mathrm{B}} \text {. }
$$

As for stratum matching, we see that there will be absolutely no bias (relative bias $=0$ ) for a perfect matching (all the members in a matched set belonging to the same population stratum), and that the bias will essentially be the same as an unmatched study (relative bias $=\mathrm{RB}^{\mathrm{B}}+$ $\mathrm{RB}^{\mathrm{E}}$ ) if the matching process by itself is no better than tossing a coin. In between these two extremes, the bias after matching will be

$$
\mathrm{RB}(\text { for stratum matching }) \approx\left(\mathrm{RB}^{\mathrm{B}}+\mathrm{RB}^{\mathrm{E}}\right) \varepsilon,
$$

where $\varepsilon(0<\varepsilon<1)$ is the error rate of the matching process. 
We can use the index of ME to quantify the effect of matching:

$$
M E=1-\frac{\mid \mathrm{RB}-(\text { for a matched study }) \mid}{\mid \mathrm{RB}(\text { for an unmatched study }) \mid} .
$$

$\mathrm{ME}=1$ indicates a perfect scenario where the bias vanishes completely after the matching. For $0<\mathrm{ME}<1$, the matching can decrease the magnitude of bias. If $\mathrm{ME}=0$, the matching doesn't help at all. The magnitude of bias remains the same as an unmatched study. $\mathrm{ME}<0$ implies the worst-case scenario where the magnitude of bias increases after the matching.

For the exposure matching, its $\mathrm{ME}$ is

$$
\mathrm{ME}(\text { for exposure matching }) \approx 1-\frac{\left|\mathrm{RB}^{\mathrm{B}}\right|}{\left|\mathrm{RB}^{\mathrm{B}}+\mathrm{RB}^{\mathrm{E}}\right|} .
$$

It can be seen that the ME can be $1,0 \sim 1,0$, or even $<0$, depending on the conditions (Table 4).

For the stratum matching, its $\mathrm{ME}$ is

$$
\mathrm{ME}(\text { for stratum matching }) \approx 1-\frac{\left|\left(\mathrm{RB}^{\mathrm{B}}+\mathrm{RB}^{\mathrm{E}}\right) \varepsilon\right|}{\left|\mathrm{RB}^{\mathrm{B}}+\mathrm{RB}^{\mathrm{E}}\right|}=1-\varepsilon .
$$

From this, one sees that stratum matching will guarantee to reduce the magnitude of bias $(0<\mathrm{ME}<1)$, irrespective of the conditions.

\section{Bias increased by exposure matching: the explanation}

Formulas presented in the previous section should help explain the rather counter-intuitive observation that the exposure matching can increase the magnitude of bias. For the exposure matching, the worst-case scenario of $\mathrm{ME}<$ 0 occurs when $r_{\mathrm{GE}} \cdot r_{\mathrm{GB}}<0$ and $\left|\mathrm{RB}^{\mathrm{E}}\right|<2 \cdot\left|\mathrm{RB}^{\mathrm{B}}\right|$ (condition VI in Table 4). In this situation, the exposure matching takes away the $\mathrm{RB}^{\mathrm{E}}$ term. But due to the opposite sign between the $R B^{B}$ and the $R B^{E}$ terms, what is left $\left(R B^{B}\right)$ is in effect larger than what is given originally $\left(R B^{B}+R B^{E}\right)$. Stated differently, the net bias of a (unmatched) study in the condition VI population is the tug of war between two opposing forces (due to stratum and due to exposure, respectively) of roughly equal measure. Take away one (by exposure matching), the scale is turned, and the magnitude of bias becomes larger.

Table 4

\begin{tabular}{|c|c|c|}
\hline \multirow[b]{2}{*}{ Conditions } & \multicolumn{2}{|l|}{ ME } \\
\hline & $\begin{array}{l}\text { Exposure } \\
\text { matching }\end{array}$ & $\begin{array}{l}\text { Stratum } \\
\text { matching }\end{array}$ \\
\hline I. $r_{\mathrm{GB}}=0$ or $\mathrm{CV}_{\mathrm{B}}=0$ & 1 & $0 \sim 1$ \\
\hline II. $\mathrm{RR}_{\mathrm{E}}=1, r_{\mathrm{GE}}=0$, or $\mathrm{CV}_{\mathrm{E}}=0$ & 0 & $0 \sim 1$ \\
\hline III. $r_{\mathrm{GE}} \cdot r_{\mathrm{GB}}>0$ & $0 \sim 1$ & $0 \sim 1$ \\
\hline IV. $r_{\mathrm{GE}} \cdot r_{\mathrm{GB}}<0$ and $\left|\mathrm{RB}^{\mathrm{E}}\right|>2 \cdot\left|\mathrm{RB}^{\mathrm{B}}\right|$ & $0 \sim 1$ & $0 \sim 1$ \\
\hline V. $r_{\mathrm{GE}} \cdot r_{\mathrm{GB}}<0$ and $\left|\mathrm{RB}^{\mathrm{E}}\right|=2 \cdot\left|\mathrm{RB}^{\mathrm{B}}\right|$ & 0 & $0 \sim 1$ \\
\hline VI. $r_{\mathrm{GE}} \cdot r_{\mathrm{GB}}<0$ and $\left|\mathrm{RB}^{\mathrm{E}}\right|<2 \cdot\left|\mathrm{RB}^{\mathrm{B}}\right|$ & $<0$ & $0 \sim 1$ \\
\hline
\end{tabular}

$\mathrm{ME}$ for the exposure matching and the stratum matching under various conditions

\section{Discussion}

We assumed that the (environmental) exposures are independent of the gene under study in each and every stratum. This is a reasonable assumption, unless of course the gene under study, beyond its possible effects on disease susceptibility, is also a gene (or in linkage disequilibrium with a gene) that influences the extent of exposure [9]. An example is alcohol-metabolizing gene. Subjects with such a gene in variant (function impaired) form may tend to have lower level of alcohol consumption, and this in turns will make those subjects less susceptible to liver cancer. In a case-control study without matching on alcohol consumption, the gene will appear to lower the risk of liver cancer, but in fact the gene may not have any "direct effect" on liver cancer risk at all. But on the other hand, even in this complicated situation one may argue against matching on alcohol consumption, if his/her main interest is on the "total effect" of the gene (including the direct effect on the disease risk and the "indirect effect" through modifying the exposure profile of a subject).

For practicing epidemiologists, our theoretical analysis in Table 4 boils down to the following two simple rules: (a) to match on anything that helps to delineate population strata and (b) to match on an exposure only when it is a strong predictor of the disease and is expected to have great variation in prevalence across population strata. For (a), this will guarantee the magnitude of bias to be smaller than that of an unmatched study, even though the stratum matching itself is crude and error prone. For (b), this would likely lead us to $\left|R B^{E}\right| \geqslant 2 \cdot\left|R B^{B}\right|$, thus avoiding the condition VI situation. A good example for exposure matching would be matching on smoking status in a lung cancer study, where the disease rate of smokers as compared to that of the nonsmokers is very high and the smoking prevalences are drastically different in different strata of the population. Although an example against exposure matching would be matching on electromagnetic field in a childhood leukemia study; the electromagnetic field exposure is a weak risk factor for childhood leukemia at best and shows little variation in prevalence if the study is conducted in a city.

In conclusion, we emphasize the importance of stratum matching and caution the uncritical use of exposure matching in a genetic association study.

\section{Acknowledgment}

This paper is partly supported by grants from the National Science Council, Taiwan, Republic of China (NSC 95-2314-B-002-242, NSC 95-3114-P-002-005-Y, and NSC 96-2314-B-002-143).

\section{References}

[1] Risch N, Merikangas K. The future of genetic studies of complex human diseases. Science 1996;273:1516-7. 
[2] Khoury MJ, Yang Q. The future of genetic studies of complex human diseases: an epidemiologic perspective. Epidemiology 1998;9:350-4.

[3] Ewens WJ, Spielman RS. The transmission/disequilibrium test: history, subdivision and admixture. Am J Hum Genet 1995;57:455-64.

[4] Witte JS, Gauderman WJ, Thomas DC. Asymptotic bias and efficiency in case-control studies of candidate genes and gene-environment interactions: basic family designs. Am J Epidemiol 1999;149: 693-705.

[5] Wacholder S, Rothman N, Caporaso N. Population stratification in epidemiologic studies of common genetic variants and cancer: quantification of bias. J Natl Cancer Inst 2000;92:1151-8.
[6] Rothman KJ, Greenland S. Modern epidemiology. 2nd edition. Philadelphia, PA: Lippincott-Raven Publishers; 1998.

[7] Lee WC. Case-control association studies with matching and genomic controlling. Genet Epidemiol 2004;27:1-13.

[8] Lee WC, Wang LY. Simple formulas for gauging the potential impacts of population stratification bias. Am J Epidemiol 2008;167:86-9.

[9] Lee WC, Chang CH. Assessing effects of disease genes and gene-environment interactions using the case-spouse design. J Epidemiol Community Health 2006;60:683-5.

[10] Agresti A. Categorical data analysis. New York, NY: John Wiley \& Sons; 1990. 